\title{
Enzyme immunoassays for the detection of microbial antigens in body fluids: an overview
}

\author{
Robert H. Yolken \\ Johns Hopkins University School of Medicine, Baltimore, Maryland, U.S.A.
}

\begin{abstract}
Traditionally, the diagnosis of infectious diseases has been accomplished by the isolation of the infecting microorganisms in pure culture. While classical cultivation systems are widely utilized for microbial diagnosis they suffer from a number of limitations. The most important limitation is that an extended period of time is of ten required before an infecting organism can be grown in sufficient quantitites so that it can be recognized and identified. This is particularly a problem in the case of viruses and slow growing bacteria where the time required for growth and specific identification can be sufficiently long so that the information derived from such cultivation is not of use to the clinician caring for the patient or to the infection control officer at tempting to prevent the spread of the infection within an institution. In addition, the reagents and equipment required for cultivation restrict the performance of microbiological techniques to laboratory situations. Diagnostic environments are particularly limited in the case of viral diagnosis since viruses require the availability of primary cells or continuous cell lines as well as sterile areas and reagents. These requirements limit viral diagnosis to central laboratory facilities and research laboratories (1-4).
\end{abstract}

For these reasons, there has been interest in the development of microbial detection techniques which would not require the prior cultivation of the organisms. Ideally, such techniques would allow for the direct detection of microorganisms in body fluids without the need for extensive sample preparation or equipment. It has long been recognized that most microbial pathogens contain antigenic determinants which allow them to be detected in body fluids and be distinguished from other microorganisms by means of specific antigen-antibody reactions. For this reason a great deal of effort has been directed at the development of assay systems capable of specific immunological detection of microbial antigens in body fluids of human and other animals. Recent advances in the successful application of immunoassay systems such as radioimmunoassay, fluorescent immunoassays and enzyme immunoassays for the detection of drugs and hormones in body fluids $(5-8)$ has encouraged investigators to apply the techniques for the detection of microbial antigens in body fluids. While many of the principles involved in the detection of microbial organisms are similar to those which are utilized for the detection of hormones or drugs in body fluids, it should be noted that there are some differences inherent in the detection of microbial antigens. First of all, microbial antigens arevirtually all polymeric often having molecular weights in excess of 200,000. Thus, many of the immunoassay detection and separation techniques which have been highly successful for the detection of smaller molecules are generally not applicable to the detection of microbial antigens in body fluids. In addition, while many microbial antigens have been well characterized in their native state, the form of the antigen which is actually present in the body fluid is often not known. Thus, while immunoassays for drugs or hormones can be devised utilizing highly specific reagents with defined immunoreactivity, it is often necessary to devise immunoassays for microbial antigens which react with a large number of different determinants to insure reaction with the form of the antigen which is expressed during the course of infection. This is particularly problematic in the case of monoclonal antibodies since such antibodies are directed only at single determinants (9-10). Care must thus be taken to ensure that the monoclonal antibodies are capable of reacting with determinants which are actually expressed during the course of infection. Another problem inherent in the detection of microbial antigens is that virtually all infectious agents are antigenic to the host and thus result in the generation of an endogenous immune response. This immune response can lead to the binding of reactive sites on the microbial antigens with endogenous antibody thus decreasing the number of sites which are available to assay immunoreactants (1). Care must be utilized to ensure that sufficient antigenic sites are exposed to allow for efficient detection of the infecting antigen.

This factor, in addition to problems associated with the production and purification of large amounts of polymeric antigens makes it difficult to utilize the competitive, labelled antigen techniques which are widely utilized for the detection of chemically defined drugs and hormones $(1,7,8,9)$. For this reason, immunoassays for the detection of microbial antigens generally utilize formats which do not require labelled antigens but rather which utilize the labelling or measurement of 
immunoglobulins. Such immunoglobulins can be prepared in large concentrations by immunization of animals with purified antigens and by monoclonal antibody techniques.

An additional constraint for the utilization of immunoassays for the detection of microbial antigens is that such antigens are of ten present in very low concentrations of body fluids. Considering that a single bacterium can weigh as little as $10^{-13} \mathrm{gm}$ and a single viral particle as little as $10^{-16} \mathrm{gms}$, infections are often accompanied by concentrations of antigens less than 1 nanogram $/ \mathrm{ml}$. Thus, immunoassays designed to measure drugs or hormones in the range of 100 nanogram $/ \mathrm{ml}$ or greater are generally not sufficiently sensitive for direct detection of microbial antigens in body fluids.

In light of these constraints, the immunoassay systems which have been most widely used for the direct detection of microbial antigens in body fluids have been ones with a high degree of sensitivity and ones which can make efficient use of labelled immunoreagents. While numerous forms of immunoassays can be designed to meet these criteria, the ones which have at tained the most widespread use have been solid phase immunoassays. These immunoassays make use of immunoreactants bound to solid phase surfaces. The presence of an antigen in a body fluid is manifested by reaction of the antigen in the body fluid with solid phase antibody and, following removal of unreacted material by washing, the subsequent addition of a labelled immunoreactant. The performance of the reactions in this way generally allows for an adequate level of sensitivity due to favorable reaction kinetics and also allows for the measurement of the labelled reaction without interference from material present in body fluids.

Numerous labels can be utilized in such solid phase reaction systems. One group of labels which allows for the formation of immunaossays with the necessary sensitivity are radioisotopes such as I125 $(11,12)$. Such isotopes can be linked to immunoglobulin molecules by a number of conjugation methods and detected in low concentrations by means of widely available gamma measurement instrumentation. In fact, radioimmunoassays have been developed for a number of microbial antigens in body fluids and have proven to be quite useful for that purpose (10-12). However, there are a number of limitations inherent in the use of radioactivity as immunoglobulin markers $(13,14)$. The principal limitations are related to the fact that the radioactive isotopes are inherently unstable, thus necessitating frequent relabelling. In addition, there is some degree of biohazard associated with the use of radioactive isotopes thus limiting the performance of radioimmune assay procedures to central laboratories. For this reason, there has been interest in the development of non-isotopic methods for the measurement of antigen-antibody reactions in body fluids. One of the most useful has been the use of enzymatic markers in the form of solid phase enzyme immunoassay systems. In such systems, molecules with enzymatic activity are utilized to bind one of the components in the antigen-antibody reaction $(1,15-17)$. The presence of the enzyme labelled immunoreactant is manifested by the addition of specific substrate which is converted to a measurable product in the presence of the bound enzyme. Since small concentrations of enzyme can catalyze the production of large amounts of product, the use of enzymes has the advantage of magnifying the initial antigen-antibody reaction and thus allowing for the detection of small amounts of antigen by the use of substrates which generate visible color. The visable color can be seen with the naked eye or be quantitated by the use of readily available colorimetric instrumentation. This allows for the performance of these assays on either a quantitative or qualitative basis.

Enzyme immunoassays can be performed in a number of reaction formats. Most enzyme immunoassays for the detection of antigens in clinical specimens are performed in a non-competitive system which consists of the binding of antibody directed at the antigen to be measured to antigen which has been bound to a solid phase surface. While numerous solid phase surfaces can be utilized for this purpose, most enzyme immunoassays utilize plastic surfaces which are capable of binding to immunoglobulins by either covalent or hydrophobic interactions (18-20). Following removal of unreacted antigen by washing, antibody directed at the antigen is added. This will bind to reactive sites available on the solid phase antigen. In the direct form of the immunoassay this second antibody is covalently coupled to an active enzyme such as horseradish peroxidase, alkaline phosphotase, betagalactosidase, or beta-lactamase (Figure 1) (21-24). The reaction is subsequently completed by removal of unreacted enzyme labelled antibody by washing and reaction with the appropriate enzyme substrate. Such direct immunoassays have the advantage of requiring few reaction steps and being relatively simple to perform. However, such direct assays do require the availability of distinct enzyme labelled reagents for each antigen to be tested. For this reason, numerous indirect formats have been devised in which unlabelled second antibody is utilized and the reactions are completed by further reaction with another enzyme labelled species (Figure 2). In many forms of indirect assays, this enzyme labelled species is an anti-immunoglobulin directed at the immunoglobulin species of second antibody. For example, enzyme labelled antibody to rabbit immunoglobulin can be utilized to complete a reaction in which rabbit immunogloublin was utilized as the second antibody. Such antiimmunoglobulin systems have the advantage that efficient enzyme labelled antiglobulins are widely available from commercial sources and generally applicable to a wide range of immunoassay systems. However, they suffer from the disadvantage that some degree of non-specific interaction can occur between the enzyme labelled antiglobulin and heterologous immunoglobulin present in the clinical specimens or utilized as the solid phase antibody. This problem can be overcome by the use of indirect systems which utilize haptenes linked to the second antibody (Figure 3). In such cases, the reactions can be completed by immunoreactants capable of efficiently binding to the haptenes. There are numerous haptene-antihaptene systems which can be utilized for this purpose. However, 
1<smiles>[3H]C[13CH][13CH]</smiles>

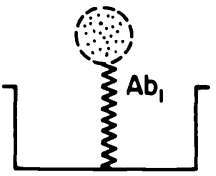

3
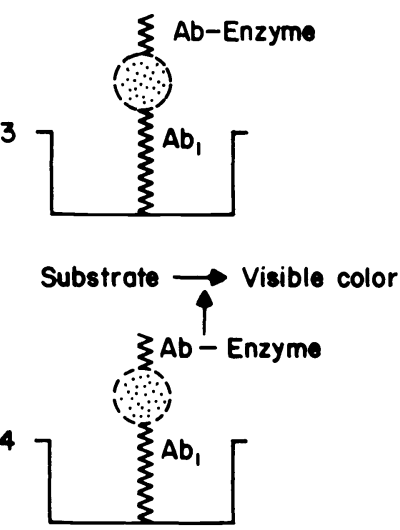

Fig. 1. Direct immunoassay system for rotavirus (Reprinted from Ref. 1). Direct enzyme-linked immunosorbent assay (ELISA) for antigen measurement. $I$ : Antibody $\left(\mathrm{Ab}_{1}\right)$ directed against the antigen to be measured is adhered to the well of a microtiter plate. 2: The test material is added; any antigen to which the antibody is directed will adhere. 3: Antibody labeled with an enzyme is added; this antibody-enzyme complex will react with the antigen that is adhered to $A b_{1}$. 4: $A$ substrate is added. The enzyme adhered to the well will convert the substrate to a visible form. The amount of color measured is proportional to the amount of antigen in the test material.

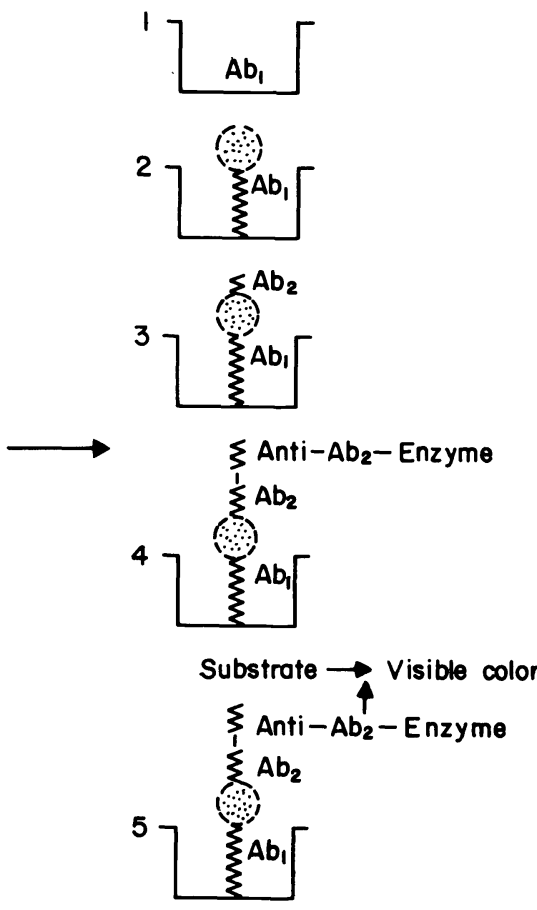

Fig. 3. Anti-haptene immunoassay system. An enzyme immunoassay is performed which utilizes an enzyme $\left(E_{1}\right)$ to generate a substrate product $\left(P_{1}\right)$. This product is then measured in a second enzyme immunoassay which utilizes an antibody to $\mathrm{P}-1$ $\left(A b_{P} 1\right)$ which is labeled with a second enzyme $\left(E_{2}\right)$. This assay results in the generation of a second reaction product $\left(\mathrm{P}_{2}\right)$ which is measured by a standard colorimetric reaction. The amount of $P_{2}$ generated by this reaction system is proportional to the product of the turnover rates of $E_{1}$ and $E_{2}\left(E_{1} \times E_{2}\right)$. 
the one which has been most widely utilized is the avidin-biotin system (24-26). In such systems the immunoglobulin is covalently linked with biotin. While there are numerous methods of conjugating biotin to immunoglobulin, this linkage can be most easily accomplished by reaction of immunoglobulin with a molar excess of biotin-n-hydroxysuccimide ester in an alkaline buffer and the removal of unreacted ester by dialysis. The immunoassay is completed by interaction with avidin which is either covalently labelled which enzyme or which is linked to enzyme by reaction with biotinillated forms of the enzyme. The avidin-biotin immunoassays can take advantage of the extremely high affinity of avidin for biotin and the fact that each molecule of avidin is capable of reacting with 4 molecules of biotin, thus providing some degree of magnification in the immunoassay system.

Enzyme immunoassays have been formulated by the above methods to detect large number of bacterial, viral, fungal, and parasitic antigens in clinical specimens $(1,21,24,26)$. Assays utilizing efficient antibodies can generally detect as little as .l nanogram of antigen directly in clinical specimens in a short period of time. This provides a sufficient level of sensitivity for the detection of many infectious diseases. However, there are also numerous infections in which antigens might be present in levels below this concentration. The efficient diagnosis of these diseases will thus require additional sensitivity to allow for their efficient detection. There are thus efforts being directed at increasing the practical sensitivity of enzyme immunoassay systems. Such efforts have been directed at the use of high energy substrates to allow for the detection of smaller amounts of enzyme, the use of enzyme cascade systems to allow for the more efficient turnover of substrate, and the use of solid phase systems which allow for the higher concentrations of immunoreactants (27-30). In addition, efforts have been directed at increasing the speed of performance of enzyme immunoassays so that they can be more efficiently utilized for the rapid diagnosis of infections. Practical methods of increasing the rapidity of enzyme immunoassays include the utilization of more efficient enzyme substrate reactions as discussed above as well as the use of reaction formats which require fewer incubation and washing steps (31). Successful application of these methods of improving the sensitivity and rapidity of enzyme immunoassays will markedly increase the utility of solid phase enzyme immunoassays and allow for their application to the diagnosis of a wide range of infectious diseases under a number of clinical, laboratory and epidemiological settings.

References

1. R.H. Yolken, Rev. Infect. Dis. 4, 35 (1982).

2. K. McIntosh, C. Wilfert, M. Chernesky, et al, J. Infect. Dis, 138, 414 (1978).

3. P.S. Gardner, Experientia 33, 1674 (1977).

4. J.W. Walsh, R. Yalow, and S.A. Berson, J. Infect. Dis. 31, 550 (1970).

5. E. Sioni and I. Hemmila, Clin. Chem, 25, 353 (1979).

6. T.P. Whitehead, L.J. Kricka, and T.J.N. Carter, Clin. Chem. 25, 1531 (1979).

7. R.H. Forsman, R.C. McCarthy, H. Markowitz, et al, Clin. Chem, 26, 1028 (1980).

8. S. Iwasha, H. Veno, T., Miya, et al, J. Biochem. 86, 943 (1979).

9. G. Kohler and C. Milstein, Nature 256, 495 (1975).

10. W. Gerhard, C.M. Croce, D. Lopes, et al, Proc. Natl. Acad. Sci. U.S.A. 75, 1510 (1978).

11. J.P. Felber, Adv. Clin. Chem, 20, 129 (1978).

12. A.E. Herner, and J.A. Clayton-Hopkins, Clin. Chem. 20, 129 (1978).

13. J.D. Rosenthal, K. Hayashi, and A.L. Notkins, Applied Microbiol. 25, 567 (1973).

14. N.H. Levitt, H.V. Miller, and G.A. Eddy, J. Clin. Microbiol. 4, 382 (1976).

15. E. Engvall, and P. Perlmann, J. Immunol. 109, 129 (1972).

16. S. Avrameas and T. Ternynck, Immunochemistry 8, 1175 (1971).

17. R.H. Yolken, H.W. Kim, T. Clem, et al, Lancet 2, 263 (1977).

18. J.E. Herrmann and M.F. Collins, J. Immunol. Methods 10, 363 (1976).

19. J.L. Guesdon and S. Avrameas, Immunochemistry 14, 443 (1977).

20. R.M. Hendry and J.E. Herrmann, J. Immunol. Methods 35, 285 (1980).

21. R.H. Yolken and P.J. Stopa, J. Clin. Microbiol. 11, 546 (1980).

22. M.J. O'Sullivan, E. Gnemmi, D. Morris, et al, Anal. Biochem. 100, 100 (1979).

23. P.K. Nakane and A. Kawaoi, J. Histochem. Cytochem. 22, 1084 (1974).

24. R.H Yolken and S.B. Wee, J. Clin. Microbiol. 19(3), 356 (1984).

25. J.L. Guesdon, T. Ternynck and S. Avrameas, J. Histochem. Cytochem. 27 1131, (197(0.

26. R.H. Yolken, F.J. Leister, L.S. Whitcomb and M. Santosham, J. Immunol. Methods 56, 319 (1980).

27. K. Puget, A.M. Michelson and S. Avrameas, Anal. Biochem. 79, 447 (1977).

28. E. Konishi, S. Inasa, K. Kondo, et al, J. Clin. Microbiol. 12, 140 (1980).

29. P.E. Stanley, Academic Press, D.L. Horrock and C.T. Peng (Eds.) 607 (1971).

30. S. Iwasha, H. Veno, T. Miya, et al, J. Biochem. 86, 943 (1979).

31. R.H. Yolken and F.J. Leister, J. Clin. Microbiol. 13, 738 (1981). 